bildgebenden Verfahren wie der Echokardiographie oder nuklearmedizinischen Techniken herangezogen wurden. An dieser Stelle sei daher ausdrücklich betont, dass beim klinischen Einsatz der technisch sehr anspruchsvollen Schnittbildverfahren stets die jeweilige Expertise mit entsprechender Ausbildung und adäquater Ausstattung vor Ort berücksichtigt werden muss.

Das derzeit quantitativ größte klinische Einsatzgebiet der beiden Methoden stellt die koronare Herzerkrankung dar. In diesem Kontext besteht die größte Stärke der MRT in der Möglichkeit, zeitlich und räumlich hochaufgelöste morphologische und funktionelle Informationen $\mathrm{zu}$ integrieren, um die Frage nach dem Vorhandensein einer myokardialen Ischämie und Viabilität zu beantworten. Der größte Nutzen der CT liegt in einer der MRT überlegenen, hochauflösenden und sehr schnellen Darstellung der Herzkranzgefäße. Aus der aktuell teilweise sehr kontrovers geführten Diskussion über diese grundsätzlich unterschiedlichen diagnostischen Ansätze (mehr funktionsbasierte gegenüber mehr angiographischer Diagnostik) wird sich in Zukunft womöglich eine differenzierte Beurteilung des Stellenwerts der beiden Methoden ergeben.

Zusammenfassend handelt es sich bei den vorliegenden Empfehlungen um eine pragmatisch sinnvolle Orientierungshilfe zum Einsatz der kardialen MRT und CT in der täglichen klinischen Praxis.

\section{Korrespondenzadresse}

\section{E. Fleck}

Klinik für Innere Medizin - Kardiologie, Deutsches Herzzentrum Berlin Augustenburger Platz 1, 13353 Berlin fleck@DHZB.de

Interessenkonflikt. Keine Angabe.

Kardiologe $2012 \cdot 6: 127 \cdot$ DOI 10.1007/s12181-012-0421-z (c) Springer-Verlag 2012

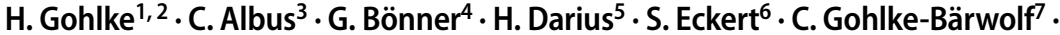
D. Gysan ${ }^{8} \cdot$ H. Hahmann ${ }^{9} \cdot$ M. Halle ${ }^{10} \cdot$ R. Hambrecht ${ }^{11} \cdot$ P. Mathes ${ }^{12} \cdot$ H.-G. Predel ${ }^{13}$. G. Sauer ${ }^{14} \cdot$ C. von Schacky ${ }^{15} \cdot$ G. Schuler ${ }^{16} \cdot$ J. Siegrist ${ }^{17} \cdot$ J. Thiery ${ }^{18} \cdot$ D. Tschöpe ${ }^{19}$. H. Völler ${ }^{20}$ - A. Wirth ${ }^{21}$

1 -, Ballrechten-Dottingen ${ }^{2}$ Herz-Zentrum, Bad Krozingen

${ }^{3}$ Klinik und Poliklinik für Psychosomatik und Psychotherapie, Universitätsklinikum, Köln

${ }^{4}$ Park-Klinikum, Bad Krozingen; ${ }^{5}$ Klinik f.Kardiologie, Angiologie u. Intensivmedizin, Vivantes Klinikum Neukölln, Berlin; ${ }^{6}$ Herz- und Diabeteszentrum Nordrhein Westfalen, Bad Oeynhausen; ${ }^{7}$ Herz-Zentrum, Bad Krozingen; ${ }^{8}$ Kardiologisch-Angiologische Gemeinschaftspraxis, Köln-Poll; ${ }^{9}$ Klinik Schwabenland, IsnyNeutrauchburg; ${ }^{10}$ Präventive und Rehabilitative Sportmedizin, Klinikum rechts der Isar, Technische Universität München, München; ${ }^{11}$ Klinik f. Kardiologie im Klinikum Links der Weser, Bremen

${ }^{12}$ Kardiologische Abteilung, Rehabilitationszentrum, München; ${ }^{13}$ Deutsche Sporthochschule, Köln; ${ }^{14}$-, Miesbach; ${ }^{15}$ Abteilung Präventive Kardiologie, Ludwig-Maximilians-Universität, München; ${ }^{16}$ Klinik für Innere Medizin/Kardiologie, Herzzentrum Leipzig, Leipzig; ${ }^{17}$ Institut für Medizinsoziologie, Universitätsklinikum Düsseldorf, Düsseldorf; ${ }^{18}$ Institut für Laboratoriumsmedizin, Klinische Chemie und Molekulare Diagnostik, Universitätsklinikum Leipzig, Leipzig; ${ }^{19}$ Herz- und Diabeteszentrum Nordrhein Westfalen, Bad Oeynhausen

${ }^{20}$ Department für Rehabilitationswissenschaften, Universität Potsdam, Potsdam; ${ }^{21}$ Deutsche Adipositasgesellschaft, Bad Rothenfelde

\title{
Erratum zu: Empfehlungen der Projektgruppe Prävention der DGK zur risikoadjustierten Prävention von Herz- und Kreislauferkrankun- gen - Teil 1: Risikostratifikation und Umsetzung der Prävention
}

\author{
Kardiologe (2012) 6:63-76 \\ http://dx.doi.org/10.1007/s12181-011-0383-6
}

Im oben genannten Beitrag wurde bei den Autorenangaben der Vorname von Herrn Prof. Martin Halle falsch abgekürzt. Wir bitten um Beachtung der im Folgenden korrekt wiedergegebenen Schreibweise: M. Halle

Präventive und Rehabilitative Sportmedizin, Klinikum rechts der Isar, Technische Universität München

Die Redaktion

\section{Korrespondenzadresse}

Prof. Dr. H. Gohlke

Neue Kirchstr. 22,

79282 Ballrechten-Dottingen

h.gohlke@t-online.de 